Tropical Journal of Pharmaceutical Research January 2021; 20 (1): 211-217

ISSN: $1596-5996$ (print); 1596-9827 (electronic)

(C) Pharmacotherapy Group, Faculty of Pharmacy, University of Benin, Benin City, 300001 Nigeria.

\title{
A comparative study of the analgesic effects of sevoflurane and propofol in children following otolaryngology surgical procedures: A pilot study
}

\author{
Yanwu Wang, Fan Yao, Yulong Lin*, Shugen Xiao* \\ Department of Anaesthesiology, Central Hospital of Xiangyang (North Area), Xiangyang Hubei Province 441021, China \\ *For correspondence: Email: lian737360936992@163.com, GwendolynChapmanucl@yahoo.com; Tel/Fax: +86710 3523491 \\ Sent for review: 20 November 2019 \\ Revised accepted: 18 December 2020
}

\begin{abstract}
Purpose: To determine the analgesic effects of sevoflurane (Sev) and propofol (Pro) in children who underwent otolaryngology surgical procedures, and their post-operative conditions.

Methods: A total of 62 (ASA I or ASA II) pre-medicated children who were about to undergo otolaryngology surgical procedures were chosen and divided equally into Sev and Pro groups, with 31 patients per group. During the surgical procedure, Sev was administered via a mask, while Pro was given i.v. Each anesthesia was followed with fentanyl administration.

Results: Pain scores such as verbal rating scale (VRS) and visual analogue scale (VAS) were slightly lower in Sev group than in Pro group. However, post-operative conditions such as emergence delirium (ED) and emergence agitation (EA) were significantly elevated in Sev group, when compared to Pro group $(p<0.05)$. In addition, patients in Sev group had higher levels of hemodynamic parameters (blood pressure), and much higher number of adverse events than those in Pro group. Thus, the overall satisfaction score and recovery characteristics, i.e., hospitalization time and recovery were slightly better in Pro-anesthetized children than in those given Sev.

Conclusion: These results suggest that except for pain score, Pro-anesthetized children fared better in terms of speedy recovery and reduced adverse effects than those given Pro. Thus, Pro may be recommended as general anaesthetic for children undergoing otolaryngology surgical procedures.
\end{abstract}

Keywords: Sevoflurane, Propofol, Pain score, Emergence agitation, Otolaryngology

\begin{abstract}
This is an Open Access article that uses a fund-ing model which does not charge readers or their institutions for access and distributed under the terms of the Creative Commons Attribution License (http://creativecommons.org/licenses/by/4.0) and the Budapest Open Access Initiative (http://www.budapestopenaccessinitiative.org/read), which permit unrestricted use, distribution, and reproduction in any medium, provided the original work is properly credited.

Tropical Journal of Pharmaceutical Research is indexed by Science Citation Index (SciSearch), Scopus, International Pharmaceutical Abstract, Chemical Abstracts, Embase, Index Copernicus, EBSCO, African Index Medicus, JournalSeek, Journal Citation Reports/Science Edition, Directory of Open Access Journals (DOAJ), African Journal Online, Bioline International, Open-J-Gate and Pharmacy Abstracts
\end{abstract}

\section{INTRODUCTION}

Several reports indicate that combination of various agents (multimodal analgesics) such as analgesics, hemodynamics, stabilizers, muscle relaxants and hypnotics in proper proportion produces adequate analgesic effect and speedy recovery, and also reduces adverse effects $[1,2]$. However, the development of an ideal multimodal anaesthetic agent with these properties from various agents at precise levels of incorporation for each surgical procedure is a challenging task to anaesthesiologists. The choice of anesthetic agent (general anesthesia) is determined by an anaesthesiologist based on patient's characteristics such as sex, age, and ASA status, as well as requirements and type of surgery [3]. 
Moreover, anaesthesiologists need to make sure that the anaesthesia of choice can improve recovery time and control pain effectively without any serious adverse effects. Pain management is an important anaesthetic care which is always debatable and challenging since it varies amongst individuals due to several factors [4]. Sevoflurane (Sev) or ultane is a poorly-soluble inhaled anaesthesia that is highly recommended as general anaesthesia for children or kids (inpatients and out-patients) because apart from its pleasant smell, it is associated with good early recovery and adequate hemodynamic stabilizing properties $[5,6]$.

However, Sev is also associated with numerous adverse effects, especially impaired cognitive function (ED/EA) and post-operative nausea and vomiting (PONV). These side effects tend to limit the use Sev since they significantly increase hospital stay and may also bother parents/caregivers and nurses/doctors due to ED-associated instability of the patients [7, 8]. On the other hand, Pro is a popular intravenous and short-acting anaesthetic agent with good recovery rate [9]. Propofol (Pro) is also used in children for its sedative effect and as general anaesthesia. It has been reported that Pro produces lower ED in children than Sev [10].

Many researchers have compared the effects of different analgesic agents (especially Sev and Pro) on pain as well as post-operative conditions such as recovery profile, ED and adverse effect in different conditions [7,11-13]. However, no comparative studies have been conducted on Sev with Pro after otolaryngology procedure in children. Therefore, the present trial was designed to compare the analgesic effects of Pro and Sev by measuring visual analogue scale (VAS) and verbal rating scale (VRS) as well as post-operative conditions such as anaesthesia emergence delirium (PAED) scale and hemodynamic parameters (blood pressure and heart rate) in children who underwent otolaryngology surgical procedures involving tonsillectomy, adenoidectomy, and ear tube (tympanostomy). The other parameters determined included satisfactory score, adverse effects as well as recovery characteristics (duration of hospital stay and recovery time).

\section{EXPERIMENTAL}

\section{Chemicals}

Propofol (Pro) and Sevoflurane (Sev) were purchased from Bayer limited, Hongkong, China.
All other chemicals and reagents used in this study were of analytical or HPLC grade.

\section{Ethical approval and recruitment of patients}

This randomized clinical trial was conducted at Central Hospital of Xiangyang (North area), China from October 2018 to March 2019. The trial was approved by the Ethical Board Committee of Central Hospital of Xiangyang (approval no. CH-X-124-234) and all the protocols used were in accordance with the guidelines of the Declaration of Helsinki (revised 1998) [4]. Parental consent was obtained from the parents of each child after explaining the details of this study. The patients included in the study were children who were about to undergo otolaryngology surgical procedures such as tonsillectomy, adenoidectomy, and tympanostomy (ear tube) without any renal, hepatic or cardiac disorders. The excluded patients were children with bleeding disorders, psychiatric illness, immunodeficiency, developmental delay, sleep apnoea and history of allergy to any type of anaesthesia or sedation drugs, as well as possible neoplasia or chronic metabolic disorders. All the children were recruited for this study through flyers and newspaper advertisements, as well as hospital electronic medical database. Initially, 75 children were screened based on the inclusion/exclusion criteria, out of which 62 children were finally included in this study.

\section{Experimental grouping and anaesthetic procedure}

A total of 62 (ASA I or ASA II) pre-medicated children (aged between 2 and 7 years) who were about to undergo otolaryngology surgical procedures were chosen and randomly divided equally into 2 groups i.e. Sev $(n=31)$ and Pro ( $n$ $=31$ ). Sevoflurane (Sev) was maintained at a minimal alveolar concentration $(3 \mu \mathrm{g}$ and $4-8$ $\%)$ via laryngeal mask by mixing with nitrous oxide and oxygen $\left(70 \% \mathrm{~N}_{2} \mathrm{O}\right.$ and $\left.30 \% \mathrm{O}_{2}\right)$ for patients in Sev group. Children in the Pro group received continuous infusion of Pro (via i.v catheter with controlled infusion-bolus) at a concentration of 2-4 mg/kg for $15 \mathrm{~min}$, which was maintained at rate of $5-8 \mathrm{mg} / \mathrm{kg} / \mathrm{h}$. Then, children in both groups were infused i.v. with fentanyl at a dose of $3 \mu \mathrm{g} / \mathrm{kg}$ during otolaryngology surgical procedures. Before the surgical procedures, the patients received oral atropine and midazolam so as to lower ED and pain. Throughout the surgical procedures, all vital signs were monitored and maintained at normal levels (using ventilation system, ECG, endtidal 
$\mathrm{CO}_{2}$ ). At the end of the surgical procedure, anaesthesia was stopped and the children were transferred to recovery unit or ward for monitoring. When the children completely recovered from anaesthesia (the time taken for recovery in each group was noted), the nurses and physicians measured pain score with respect to VRS and VAS by asking the patients relevant questions. The pain score was measured $5 \mathrm{~min}$ after the patients opened their eyes. Finally, oral acetaminophen was given to reduce postoperative pain in children who still felt pain. The patients were discharged after $4-6 \mathrm{~h}$ to resume their normal life activities.

\section{Assessment of post-operative conditions}

The nurses or resident anesthesiologists in the recovery room or post-operative unit recorded the duration and levels of ED (mild, moderated or high) using Pediatric Anesthesia Emergence Delirium (PAED) score. Emergence Delirium (EWD) was assessed on a four-point scale [8]. However, only few children encountered ED, and this was controlled by injecting an antipsychotic drug (benzodiazepine). The recovery time (time from surgery to complete recovery i.e. eye opening time and time it took for the patient to become responsive) were recorded. The overall hospital stay for each child was also recorded. Moreover, hemodynamic parameters such as diastolic blood pressure (DBP), mean arterial blood pressure (MAP), systolic blood pressure (SBP) and heart rate (HR) were monitored during surgical procedure and recorded only in the recovery room (5 min after opening of eyes). The incidence of adverse events such as vomiting and nausea (PONV), laryngospasm, headache, drowsiness, and bronchospasm were also recorded. Finally, after recovery and before discharge, satisfaction score was determined based on pain score and response from each patient in the recovery room. During this study, 2 children from Sev group were excluded due to lack of insufficient anaesthesia. Hence, only 18 children in Sev completed the study.

\section{Statistical analysis}

Data are expressed as mean \pm standard deviation (SD). Differences between the two groups (Sev vs Pro) were analysed using an independent student $t$-test, followed by one-way analysis of variance (ANOVA). All statistical analyses were done with SPSS software (version 21; IBM, USA). The pain scores were analysed using Mann-Whitney U-test. Values of $p$ less than 0.05 were considered statistically significant.

\section{RESULTS}

\section{Demographic data}

Table 1 shows the demographic profiles of children who underwent otolaryngology surgical procedures in the Sev and Pro groups, indicating gender, ASA status, age, weight, height and type of surgery. Comparison of the Sev and Pro groups revealed no significant difference in any demographical data. The post-operative pain score/scale was evaluated in terms of visual analogue scale (VAS) and verbal rating scale (VRS).

Table 1: Comparison of demographic profiles of children who underwent otolaryngology surgical procedures in the Sev and Pro groups

\begin{tabular}{lcc}
\hline Parameter & Sev $\mathbf{( n = 2 0 )}$ & Pro $(\mathbf{n = 2 0})$ \\
\hline Gender (male/female) & $8 / 12$ & $9 / 11$ \\
ASA status (I/II) & $15 / 5$ & $13 / 7$ \\
Age (years) & $4.10 \pm 0.90$ & $4.20 \pm 1.05$ \\
Weight $(\mathrm{kg})$ & $18.90 \pm 1.25$ & $19.30 \pm 1.10$ \\
Height $(\mathrm{cm})$ & $105.27 \pm 10.00$ & $102.00 \pm 12.80$ \\
Type of surgery & $2 / 12 / 6$ & $1 / 14 / 5$ \\
$(\mathrm{~A} / \mathrm{T} / \mathrm{ET})^{\dagger}$ & &
\end{tabular}

Data are expressed as mean \pm standard deviation (SD). ${ }^{\dagger} \mathrm{A}$ : adenoidectomy, $\mathrm{T}$ : tonsillectomy, $\mathrm{ET}$ : ear tube (tympanostomy), ASA: American Society of Anaesthesiologists

\section{Post-operative pain score and ED}

Figure 1 shows the post-operative pain score (VRS and VAS) of children who underwent otolaryngology surgical procedures in the Sev and Pro groups. Values of VRS and VAS were significantly higher in Pro group than in Sev group $(p<0.05)$. Thus, Sev produced better analgesic effect than Pro. The values of postoperative ED at 10 and $30 \mathrm{~min}$ in Sevanaesthetized children were significantly higher than the corresponding values in children anaesthetized with Pro $(p<0.05$; Figure 2). In both groups, values of ED at 10 min were higher than those at $30 \mathrm{~min}$, which indicate that with time, the anaesthetic effect decreased.

\section{Satisfaction score}

Figure 3 shows the satisfaction score (after recovery and before discharge) of children who underwent otolaryngology surgical procedures in Sev and Pro groups. Both groups had equal levels of satisfaction score (no significant changes between them), but patients in the Pro 
group had marginally better satisfaction score than those in Sev group.

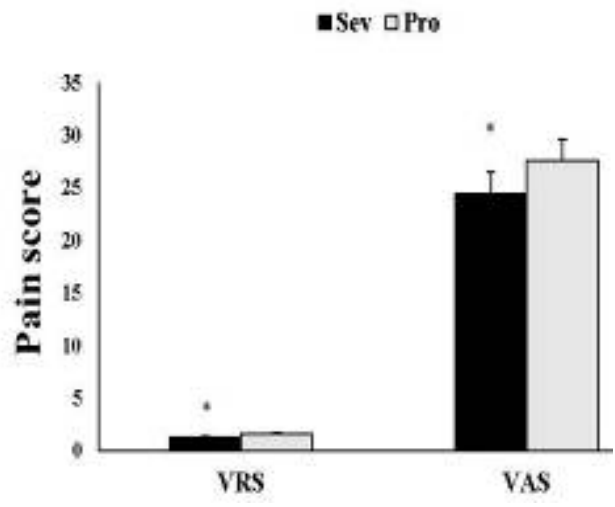

Figure 1: Post-operative pain score (VRS and VAS) of children who underwent otolaryngology surgical procedures under Sev or Pro anaesthesia. Data are expressed as mean \pm SD. ${ }^{\star} P<0.05,{ }^{\#} p<0.01$; Sev vs. Pro

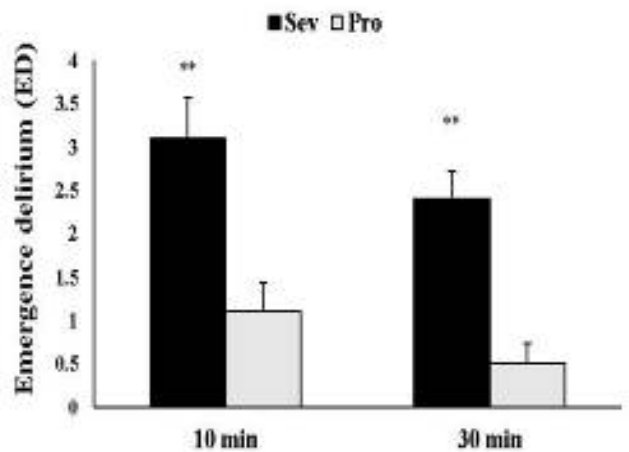

Figure 2: Levels of post-operative emergence delirium (ED) of children who underwent otolaryngology surgical procedures in the Sev- and Pro-treated groups. Data are expressed as mean \pm SD. ${ }^{\star} P<0.05$, ${ }^{\sharp} p<0.01$; Sev vs Pro

\section{Hemodynamic parameters}

The hemodynamic parameters (post-operative blood pressure and heart rate) in the Sev and Pro groups are shown on Table 2. Children in both groups had normal heart rate and blood pressure (DAP, SAP and MAP). Nevertheless, Sev-anesthetized children had significantly increased blood pressure, relative to those in Pro-anesthetized group $(p<0.05)$. There was no statistically significant difference in heart rate between the Sev and Pro groups.

\section{Adverse events recovery time and duration of hospital stay}

The various post-operative adverse events as well as recovery time (Table 3 ) and duration of hospital stay (Table 4) in children who underwent otolaryngology surgical procedures under Sev and Pro anaesthesia are provided below. There

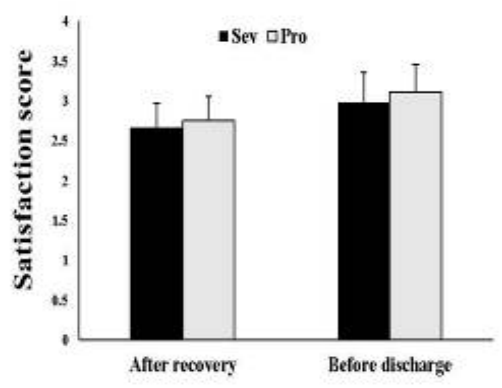

Figure 3: Satisfaction score (after recovery and before discharge) of children who underwent otolaryngology surgical procedures in the Sev and Pro groups. Data are expressed as mean \pm standard deviation (SD). ${ }^{*} p$ $<0.05 ;{ }^{\#} p<0.01$; Sev vs Pro

Table 2: Hemodynamic parameters (post-operative blood pressure and heart rate) in Sev and Pro groups of children who underwent otolaryngology surgical procedures

\begin{tabular}{lcc}
\hline Parameter & Sev $(\mathbf{n}=\mathbf{2 0})$ & Pro $(\mathbf{n = 2 0})$ \\
\hline DAP $(\mathrm{mmHg})$ & $86.20 \pm 4.15^{*}$ & $82.40 \pm 3.80$ \\
SAP $(\mathrm{mmHg})$ & $128.33 \pm 5.50^{*}$ & $120.70 \pm 6.05$ \\
MAP $(\mathrm{mmHg})$ & $107.08 \pm 5.22^{*}$ & $102.74 \pm 5.13$ \\
HR (beat/min) & $80.12 \pm 4.32$ & $81.12 \pm 4.32$ \\
\hline Data are expressed as mean \pm standard deviation \\
(SD). ${ }^{*} P<0.05 ;{ }^{\sharp} p<0.01$; Sev vs Pro. HR: heart rate; \\
DAP: diastolic arterial pressure; SAP: systolic arterial \\
pressure; MAP: mean arterial pressure
\end{tabular}

Table 3: Post-operative adverse events in children who underwent otolaryngology surgical procedures in Sev and Pro groups

\begin{tabular}{lcc}
\hline Adverse effect & Sev $(\mathbf{n = 2 0})$ & Pro $(\mathbf{n}=\mathbf{2 0})$ \\
\hline PONV & $5(25)^{\star *}$ & $1(5)$ \\
Bronchospasm & - & - \\
Laryngospasm & $2(10)^{\star}$ & - \\
Dizziness & - & - \\
Headache & $1(5)$ & $3(15)^{\star}$ \\
\hline Data are shown as number of patients (n) and $\% .{ }^{\star} P<$ \\
$0.05 ;{ }^{*} p<0.01$; Sev vs. Pro
\end{tabular}

were higher incidence of adverse events (PONV and laryngospasm) in Sev group than in Pro group, whereas cases of headache were higher in the Pro group. Due to higher cases of adverse effects and ED in Sev group, the hospital stay and recovery time were longer in this group. The duration of hospital stay and recovery time were shorter in the Pro group, when compared to the Sev group. Thus, Pro produced moderate analgesic effect, better recovery time and lower incidents of adverse effects.

\section{DISCUSSION}

Sevoflurane (Sev) and propofol (Pro) are used as general anaesthesia during various paediatric surgical procedures [11,12]. However, each of these anaesthetic agents has its merits and 
Table 4: Recovery time and hospital stay (time) in children who underwent otolaryngology surgical procedures in Sev and Pro groups

\begin{tabular}{lcc}
\hline Parameter & Sev $(\mathbf{n}=\mathbf{2 0})$ & Pro $(\mathbf{n = 2 0})$ \\
\hline Recovery time $(\min )$ & $85.20 \pm 4.15^{*}$ & $82.40 \pm 3.80$ \\
Hospital stay (min) & $244.10 \pm 10.50^{*}$ & $228.80 \pm 8.05$ \\
\hline Data are expressed as mean \pm standard deviation \\
(SD); ${ }^{*} p<0.05 ;{ }^{\#} p<0.01$, Sev vs Pro
\end{tabular}

demerits which formed the basis of the present study aimed at comparison of their analgesic and postoperative complications in children who underwent otolaryngology surgical procedures. Thus, this clinical trial was designed to compare the analgesic effects of the two agents (Pro and Sev) by measuring VAS, VRS and post-operative conditions such as ED, hemodynamic parameters, satisfaction score, and adverse effects, as well as recovery time. Children anesthetized with Sev experienced better analgesic effect, but Pro-anesthetized children had lower incidents of adverse effects (ED and PONV) and shorter recovery time.

The best way to assess pain after various surgical procedures is by evaluating pain score (VAS and VRS). Post-operative pain score was significantly higher in Pro group than in Sev group. Similarly, Fassoulaki and his co-workers [14] have reported that Sev produced lower VAS score than Pro or desflurane, although the difference was not significant, unlike the results obtained in this study. The variation in results might be due to differences in the doses of Sev and Pro, as well as differences in surgical procedures used in both studies. Overall, Sev group showed better analgesic effect than Pro group due to lower oxygen saturation property of Sev. Emergence delirium (ED) is one of the major adverse effects seen in various general anaesthesias. Moreover, ED enhances the risk of self-injury and extended hospital stay, making it a major post-surgery concern [15]. Hence, in this study, ED score (PAED score) was determined using Aono Scaling method [8]. Many researchers have reported that $\mathrm{Sev}$ is highly associated with various adverse effects in children, especially ED $[16,17]$. In this study, the values of post-operative ED at 10 and $30 \mathrm{~min}$ in Sev-anesthetized children were significantly higher than those in Pro-anesthetized children. Smith and co-workers [18] have reported that increased incidence of ED after Sev administration might be due to low blood gas solubility. Nevertheless, the exact reason for increased ED in Sev group is largely unknown [16]. The incidence of ED declines with time, indicating clearly that the anaesthetic effect of Sev might indirectly contribute to ED.
Children are highly susceptible to blood pressure changes after any kind of anaesthesia. Therefore, changes in blood pressure and heart rate after general anaesthesia with Sev and Pro were determined in this study. Post-operative hemodynamic parameters such as blood pressure (DAP, SAP, and MAP) and heart rate were normal even after Sev or Pro anaesthesia. However, children in Sev group had higher blood pressure than those in Pro group. There was no statistically significant difference in heart rate between Sev- and Pro-administered children. Based on pain score, the satisfaction score was calculated on a 4-grade scale by nurses in the recovery room. Both groups showed equal levels of satisfaction score (no significant changes between each group), but the Pro group had marginally better satisfaction score than the Sev group. Similarly, Pieters and his colleagues 13] have reported that satisfaction score was similar in Sev and Pro groups. Several reports have demonstrated that post-operative nausea and vomiting (PONV), headache, spasm and dizziness are common post-anaesthesia adverse effects in children $[19,20]$. Thus, in this study, the adverse events caused by Sev and Pro were cross-checked in the children after otolaryngology surgical procedures. The incidence of PONV and laryngospasm were higher in the Sev group than in the Pro group. This result is in agreement with the results of Pieters et al [13], and Moore et al. [19]. These studies also showed that PONV and spasm were higher in Sev group than in Pro group. Finally, the duration of hospital stay and recovery time were shorter in children who received in Pro anaesthesia than in those who were given Sev. This may be due to the lower cases of adverse events and ED in the Pro group.

\section{Limitations of the study}

This study has some limitations. In the first place, there was no combination group, i.e., children given Sev + Pro. Since Sev and Pro have some advantages and disadvantages, combination anaesthesia involving the two agents might produce a better result than when Sev and Pro are used singly. Secondly, three different surgical procedures were used in this study. Since each of the surgical procedures triggers a different degree of pain, the comparison of the analgesic properties of the two agents (Sev and Pro) might be subjective.

\section{CONCLUSION}

These results indicate that Sev exhibits better analgesic effect than Pro, but Pro-anesthetized children show shorter recovery time (early 
recovery/speedy recovery), slightly reduced blood pressure and lower adverse effects, especially ED and PONV, than Sev-anesthetized children. Both Pro and Sev produce similar satisfaction scores. Thus, Pro might be a better choice than Sev as general anaesthesia for children undergoing otolaryngology surgical procedures. However, more clinical trials are needed to confirm this conclusion.

\section{DECLARATIONS}

\section{Acknowledgement}

This study was funded by Central Hospital of Xiangyang as a startup fund.

\section{Conflict of interest}

No conflict of interest is associated with this study.

\section{Contribution of authors}

We declare that this work was done by the authors named in this article and all liabilities pertaining to claims relating to the content of this article will be borne by the authors. Yanwu Wang and Shugen Xiao involved in Concepting and designing this study. Fan Yao and Yulong Lin involved in data analysis as well as conducted this trial. Yulong Lin and Shugen Xiao wrote this manuscript.

\section{Open Access}

This is an Open Access article that uses a funding model which does not charge readers or their institutions for access and distributed under the terms of the Creative Commons Attribution License (http://creativecommons.org/licenses/by/ 4.0) and the Budapest Open Access Initiative (http://www.budapestopenaccessinitiative.org/rea d), which permit unrestricted use, distribution, and reproduction in any medium, provided the original work is properly credited.

\section{REFERENCES}

1. Moro ET, Leme FC, Noronha BR, Saraiva GF, de Matos Leite NV, Navarro LH. Quality of recovery from anesthesia of patients undergoing balanced or total intravenous general anesthesia. Prospective randomized clinical trial. J Clin Anesth 2016; 35: 369375.

2. Massad IM, Mohsen WA, Basha AS, Al-Zaben KR, AlMustafa MM, Alghanem SM. A balanced anesthesia with dexmedetomidine decreases postoperative nausea and vomiting after laparoscopic surgery. Saudi Med J 2009; 30: 1537-1541.

3. Siampalioti A, Karavias D, Zotou A, Kalfarentzos F, Filos $K$. Anesthesia management for the super obese: is sevoflurane superior to propofol as a sole anesthetic agent? A double-blind randomized controlled trial. Eur Rev Med Pharmacol Sci 2015; 19: 2493-2500.

4. Zhang J, Shi K, Jia H. Ketamine and bupivacaine attenuate post-operative pain following total knee arthroplasty: A randomized clinical trial. Exp Ther Med 2018; 15(6): 5537-5543.

5. Zhenqiang S, Fei S, LU L. Effects of sevoflurane and propofol on hemodynamics, cerebral oxygen metabolism and analgesia during maintenance of paediatric anaesthesia. Trop J Pharm Res 2019; 18(1): 187-92.

6. Lee CJ, Lee SE, Oh MK, Shin CM, Kim YJ, Choe YK, Cheong $\mathrm{SH}$, Lee $\mathrm{KM}$, Lee JH, Lim SH, Kim YH. The effect of propofol on emergence agitation in children receiving sevoflurane for adenotonsillectomy. Korean $\mathrm{J}$ Anesthesiol 2010; 59(2): 75.

7. Gupta A, Stierer T, Zuckerman R, Sakima N, Parker SD, Fleisher LA. Comparison of recovery profile after ambulatory anesthesia with propofol, isoflurane, sevoflurane and desflurane: a systematic review. Anesth Analg 2004; 98(3): 632-641.

8. Aono J, Ueda W, Mamiya K, Takimoto E, Manabe M. Greater incidence of delirium during recovery from sevoflurane anesthesia in preschool boys. Anesthesiology: J Am Soc Anesthesiol 1997; 87(6): 1298-1300.

9. Mohammadi SS, Nasiri AK, Shoeibi G. Effects of propofol-thiopental sodium admixture on hypnotic dose, pain on injection and hemodynamic responses during induction of anesthesia. Int J Pharm 2006; 2: 443-446.

10. Nakayama S, Furukawa $H$, Yanai H. Propofol reduces the incidence of emergence agitation in preschool-aged children as well as in school-aged children: a comparison with sevoflurane. J Anesth 2007; 21(1): 1923

11. Hasani A, Gecaj-Gashi A, Llullaku S, Jashari $H$. Postoperative analgesia in children after propofol versus sevoflurane anesthesia. Pain Med 2013; 14(3): 442-446.

12. Shah A, Adaroja, RN. Comparison of haemodynamic changes with propofol and sevoflurane anaesthesia during laparoscopic surgery. Emergence 2011; 4(5): 68.

13. Pieters BJ, Penn E, Nicklaus P, Bruegger D, Mehta B, Weatherly R. Emergence delirium and postoperative pain in children undergoing adenotonsillectomy: a comparison of propofol vs sevoflurane anesthesia. Pediatr Anesth 2010; 20(10): 944-950.

14. Fassoulaki A, Melemeni A, Paraskeva A, Siafaka I, Sarantopoulos C. Postoperative pain and analgesic requirements after anesthesia with sevoflurane, desflurane or propofol. Anesth Analg 2008; 107(5): 1715-1719.

Trop J Pharm Res, January 2021; 20(1): 216 
15. Bajwa SA, Costi D, Cyna AM. A comparison of emergence delirium scales following general anesthesia in children. Pediatr Anesth 2010; 20(8): 704-711.

16. Kanaya $A$, Kuratani $N$, Satoh D, Kurosawa S. Lower incidence of emergence agitation in children after propofol anesthesia compared with sevoflurane: a metaanalysis of randomized controlled trials. J Anesth 2014; 28(1): 4-11.

17. Uezono S, Goto $T$, Terui $K$, Ichinose $F$, Ishguro $Y$, Nakata $Y$, Morita S. Emergence agitation after sevoflurane versus propofol in pediatric patients. Anesth. Analg 2000; 91(3): 563-566.
18. Smith I, Nathanson M, White PF. Sevoflurane-a longawaited volatile anaesthetic. Br J Anaesth 1996; 76(3): 435-445.

19. Moore JK, Moore EW, Elliott RA, St Leger AS, Payne K, Kerr J. Propofol and halothane versus sevoflurane in paediatric day-case surgery: induction and recovery characteristics. Br J Anaesth 2003; 90(4): 461-466.

20. Tramer M, Moore A, McQuay H. Propofol anaesthesia and postoperative nausea and vomiting: quantitative systematic review of randomized controlled studies. $\mathrm{Br} \mathrm{J}$ Anaesth 1997; 78(3): 247-255. 